\title{
Multiple Retroperitoneal Schwannomas Mimicking Adrenal Tumors
}

\author{
Koichi Oishi $^{\mathrm{a}}$ Toshiyuki Itamoto $^{\mathrm{a}}$ Sho Okimoto $^{\mathrm{a}}$ \\ Takashi Nishisakab \\ Departments of ${ }^{\mathrm{a}}$ Surgery and ${ }^{\mathrm{b}}$ Pathology, Hiroshima Prefectural Hospital, \\ Hiroshima, Japan
}

\section{Key Words}

Retroperitoneal schwannoma $\cdot$ Computed tomography scanning $\cdot$ Adrenal tumor

\begin{abstract}
Schwannomas are benign tumors mostly arising from the head and neck. Retroperitoneal schwannomas are rare. Here we report the case of a 68-year-old woman with multiple retroperitoneal schwannomas. An abdominal computed tomography (CT) scan showed 1 non-enhancing mass with a low-density area, $4.6 \mathrm{~cm}$ in diameter, in the left adrenal region and another non-enhancing mass with a low-density area, $2.9 \mathrm{~cm}$ in diameter, in the paraaortic region. The patient had regularly undergone $\mathrm{CT}$ scans for malignant tumor screening in maintenance dialysis patients. Retrospectively, the 2 tumors had existed in the same regions in previous CT scans and had gradually increased in size. The tumors were completely resected. Pathological diagnosis of both tumors was schwannoma. When a CT scan shows a slowly growing retroperitoneal tumor with a low-density area, schwannoma should be considered. If the tumor increases in size, complete resection should be performed for pathological diagnosis.
\end{abstract}

\section{Introduction}

Schwannomas are benign tumors that arise from Schwann cells. These tumors are mostly found in the head and neck regions, and retroperitoneal schwannomas are rare. Multiple occurrences in the retroperitoneal region are extremely rare. We report on a 68-year-old woman with retroperitoneal schwannomas that were diagnosed by microscopic examination of surgically resected specimens, and we present a review of the literature on retroperitoneal schwannomas. 


\section{Case Report}

A 68-year-old woman who had been receiving hemodialysis for chronic renal failure due to diabetic nephropathy was referred to our hospital for treatment of retroperitoneal tumors. The tumors were detected by one of the abdominal computed tomography (CT) scans that she had regularly undergone. She had been diagnosed with diabetes mellitus at the age of 46 years and had received hemodialysis 3 times a week since she was 59 years of age. She had no history of symptoms of von Recklinghausen disease. There was no family history of cancer. On physical examination at the time of consultation, her body temperature was $36.6^{\circ} \mathrm{C}$, blood pressure was $164 / 80 \mathrm{~mm} \mathrm{Hg}$, and pulse rate was 67 beats/min and normal in rhythm. Her height was $160 \mathrm{~cm}$ and weight was $67 \mathrm{~kg}$. Hematocrit was $29.7 \%$ and hemoglobin level was $9.8 \mathrm{~g} / \mathrm{dl}$. In renal function tests, blood urea nitrogen was $35.6 \mathrm{mg} / \mathrm{dl}$ and serum creatinine level was $7.16 \mathrm{mg} / \mathrm{dl}$. Serum levels of sodium, potassium and chloride were 136, 6.0 and $101 \mathrm{mEq} / \mathrm{l}$, respectively. Other results of complete blood count and liver function tests performed on admission were within normal ranges. Serum levels of adrenaline, noradrenaline, dopamine, cortisol and aldosterone were within normal ranges $(24 \mathrm{pg} / \mathrm{ml}, 207 \mathrm{IU} / \mathrm{l}$, $5 \mathrm{IU} / \mathrm{l}, 12.2 \mu \mathrm{g} / \mathrm{dl}$ and $94.1 \mathrm{pg} / \mathrm{ml}$, respectively). Serum levels of carcinoembryonic antigen and carbohydrate antigen 19-9 were within normal ranges. An abdominal CT scan with administration of intravenous contrast material showed 1 non-enhancing mass with a low-density area, $4.6 \mathrm{~cm}$ in diameter, in the left adrenal region and another non-enhancing mass with a low-density area, $2.9 \mathrm{~cm}$ in diameter, in the para-aortic region ( $\underline{\mathrm{fig}} \mathbf{1} \mathrm{a}, \mathrm{d}$ ). The patient had been regularly examined by CT scan due to chronic renal failure. Retrospectively, the 2 tumors had never been pointed out by a physician but had existed in the same regions on a CT scan for 1 (fig. 1b, e) and 2 years (fig. 1c, f) before admission. The mean doubling times of the tumors in the left adrenal region and para-aortic region were 422 days and 270 days, respectively. The tumors had gradually increased in size on CT. Preoperative diagnosis was multiple pheochromocytomas or nonfunctioning adrenocortical carcinomas with lymph node metastasis, and she underwent an operation. On exploration of the intraabdominal cavity, there were 2 tumors. One was in the para-aortic region and the other was in the left adrenal region. The tumor in the left adrenal region had compressed the left normal adrenal gland but was easily detached. Both tumors were completely excised and were diagnosed as schwannomas by microscopic examination of frozen sections.

On gross examination, the tumor measuring $4.8 \mathrm{~cm}$ in diameter in the left adrenal region included yellow solid areas and cystic degeneration. The other tumor, $3.0 \mathrm{~cm}$ in diameter, in the para-aortic region included the same features (fig. 2). Microscopic examination revealed that both tumors were composed of spindle cells with blunt nuclei in a fascicular pattern. Other regions in both tumors were composed of hypocellular spindle cells in a loose myxoid matrix. In immunohistochemical examination, spindle cells reacted to $S-100$ but did not react to anti-smooth-muscle actin. These findings corresponded to multiple schwannomas ( $\underline{\text { fig. } 3 \text { ). }}$.

The patient was discharged 10 days after surgery and has had no recurrence in the 1 year since resection.

\section{Discussion}

Generally, schwannomas are benign tumors that develop from Schwann cells of the peripheral nerve sheath. These tumors occasionally occur in patients with von Recklinghausen disease. The most frequent sites are in the head and neck region. Retroperitoneal schwannomas are rare, accounting for only $1.2 \%$ of all retroperitoneal tumors [1]. The proportion of retroperitoneal schwannomas in all schwannomas has been reported to be $0.7-2.6 \%$ [2,3]. Multiple retroperitoneal schwannomas are extremely rare. In our review of the English literature, we found only 3 reports of multiple retroperitoneal schwannomas [4-6]. Li et al. [6] reported 82 cases of retroperitoneal schwannomas, 2 of which were multiple schwannomas (2.4\%). However, this statement about multiple schwannomas was the only information and there was no detailed information in their report about the age and sex of the patients, 
tumor size or location of the tumors. The other 2 reports were case reports. One was a report of a 47-year-old man with asynchronous tumors measuring 14, 16 and $10 \mathrm{~cm}$ [4]. The tumors histologically included low-grade sarcomatous change in a neurofibroma. The other was a report of a 49 -year-old man with multiple retroperitoneal schwannomas [5]. However, 1 tumor was located in the paravertebral region at the T10 spinal level that was thought to be the posterior mediastinal region. Therefore, our patient is an extremely rare case of synchronous multiple schwannomas located in the retroperitoneal region and mimicking an adrenal tumor with lymph nodes metastasis.

Schwannomas are generally solitary, well circumscribed and encapsulated [7]. Most patients are diagnosed at the age of 25-55 years, but schwannomas can occur at any age. There is no gender predilection. Most typical schwannomas grow slowly [8]. Moreover, clinical symptoms of retroperitoneal schwannomas appear later due to the anatomical position. When tumors are markedly increased in size, clinical symptoms of retroperitoneal schwannomas are initially noticed. Chief complaints of retroperitoneal schwannoma are often varied and nonspecific [7]. Some patients with retroperitoneal schwannomas present with vague, dull abdominal pain. Moreover, there are no distinctive findings to be noted in blood chemistry examination. Recently, with advances in imaging modalities, incidental detection of retroperitoneal tumors has been increasing, especially with increasing medical examination health screening. However, it is rare for retroperitoneal tumors to be preoperatively diagnosed as schwannomas. Small schwannomas appear as a homogenous, encapsulated mass, whereas large schwannomas show cystic degeneration, hemorrhage or central necrosis [9]. The most characteristic finding in schwannoma is cystic change. On CT scan with contrast enhancement, non-enhancing low-density areas in the tumor correspond to regions of cystic degeneration or necrotic regions [10]. In our case, retroperitoneal tumors were located in the left adrenal and para-aortic regions. We had preoperatively considered the tumors to be pheochromocytoma and paraganglioma or a nonfunctioning adrenal cancer with lymph node metastasis or malignant retroperitoneal tumors. CT imaging of typical adrenocortical carcinomas shows an inhomogeneous mass with heterogeneous enhancement of solid components [11]. Functional adrenocortical carcinomas are easily diagnosed by characteristic hormonerelated symptoms or blood chemistry tests. However, nonfunctioning adrenocortical carcinomas that present no characteristic symptoms are difficult to diagnose by CT findings alone. Moreover, the tumor near the abdominal aorta was suspected to be lymph node metastasis from adrenocortical carcinoma. However, CT findings in our case were inconsistent with typical types of adrenocortical carcinomas.

Additionally, nonfunctioning pheochromocytoma and paraganglioma were considered based on the location of the tumors. Characteristics of CT findings in typical pheochromocytomas are an inhomogeneous mass with relatively strong enhancement and a low-density area in tumors such as schwannomas [12]. Typical pheochromocytomas present characteristic clinical symptoms, whereas nonfunctioning pheochromocytomas commonly present no characteristic symptoms. Therefore, Inokuchi et al. [13] reported that it is difficult to differentiate between retroperitoneal schwannomas and nonfunctioning pheochromocytomas by imaging.

Consequently, a definite diagnosis can only be made histologically. The location of tumors and the existence of multiple tumors in our case made diagnosis difficult. 
However, CT imaging in the patient showed a low-density area in the tumor, retrospectively. Moreover, comparison with previous CT scans performed 1 and 2 years before showed that the tumors had been gradually increasing in size. Tumor doubling time was calculated by comparison with previous CT scans. Doubling times of most malignant tumors are less than 6 month [14]. There have been no reports on tumor doubling times of retroperitoneal schwannomas. However, tumor doubling times of vestibular schwannomas have been reported to be over 1 year [15]. Tumor doubling times in our patient were about 1 year. These findings were consistent with the characteristics of schwannomas, i.e. slow tumor growth and having cystic change. Additionally, a case of schwannoma in which a long-term course is observed is extremely rare.

In summary, we have reported a case of multiple retroperitoneal schwannomas. When a CT scan shows a slowly growing retroperitoneal tumor with a low-density area, schwannoma should be considered. If a retroperitoneal tumor is considered to be schwannoma, the patient should be carefully followed up. If the tumor gradually increases in size or clinical symptoms appear, complete surgical resection should be performed for pathological diagnosis.

\section{Disclosure Statement}

The authors have no conflicts of interest to declare. 

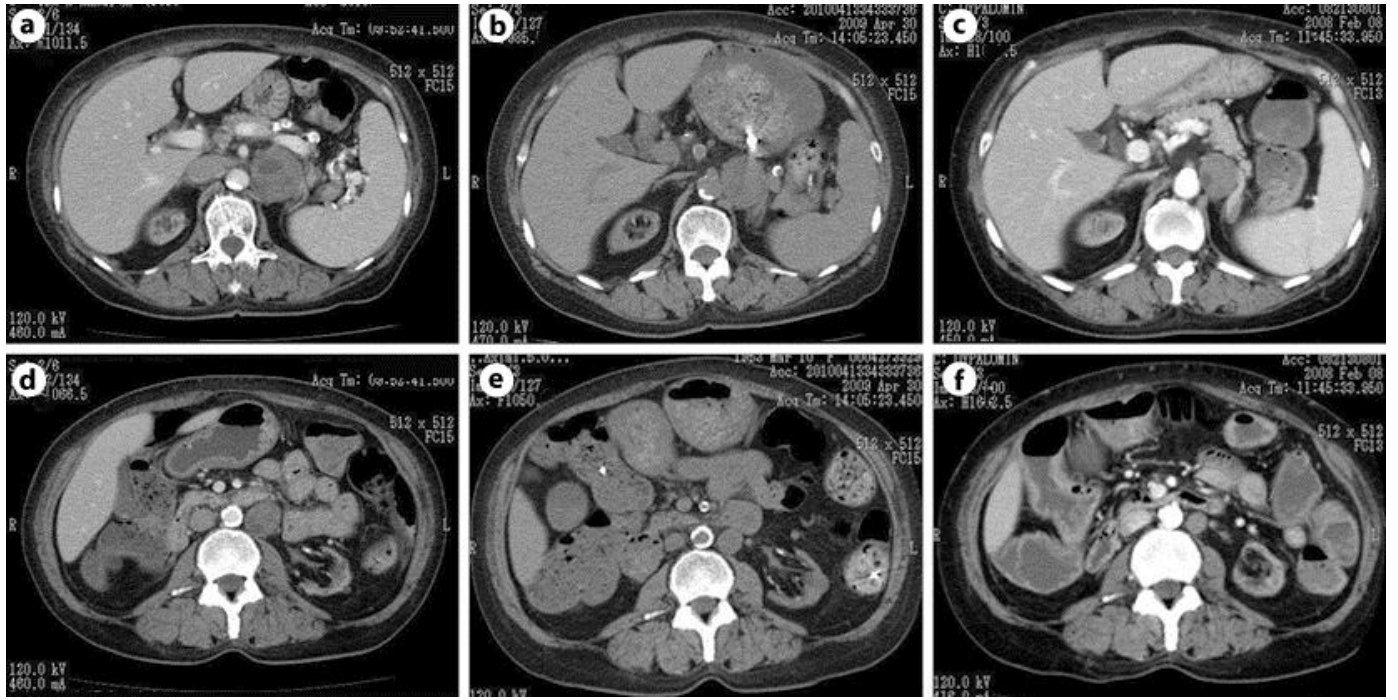

Fig. 1. CT scan of the abdomen. CT scan of the abdomen with administration of intravenous contrast material showed 1 non-enhancing mass with a low-density area, $4.6 \mathrm{~cm}$ in diameter, in the left adrenal region (a) and another non-enhancing mass with a low-density area, $2.9 \mathrm{~cm}$ in diameter, in the paraaortic region (d). The 2 tumors could be seen in the same regions on CT scans performed 1 (b, e) and 2 years before $(\mathbf{c}, \mathbf{f})$. The tumors had gradually increased in size.
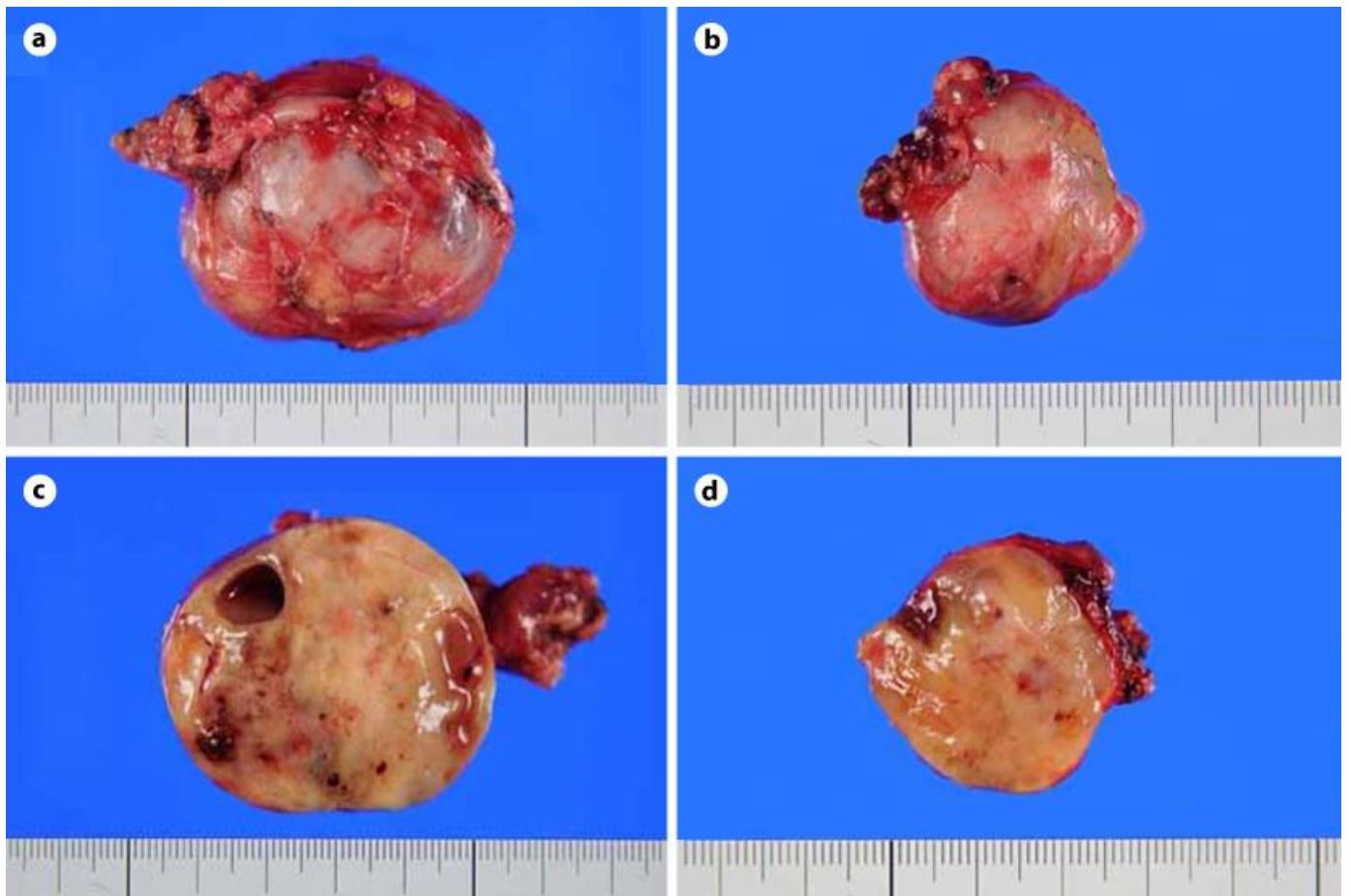

Fig. 2. Gross features of the schwannomas. The cut surface of the tumor $4.8 \mathrm{~cm}$ in diameter from the left adrenal region showed yellow solid areas and cystic degeneration $(\mathbf{a}, \mathbf{c})$, and the cut surface of the other tumor $(3.0 \mathrm{~cm}$ in diameter) from the para-aortic region showed the same features $(\mathbf{b}, \mathbf{d})$. 

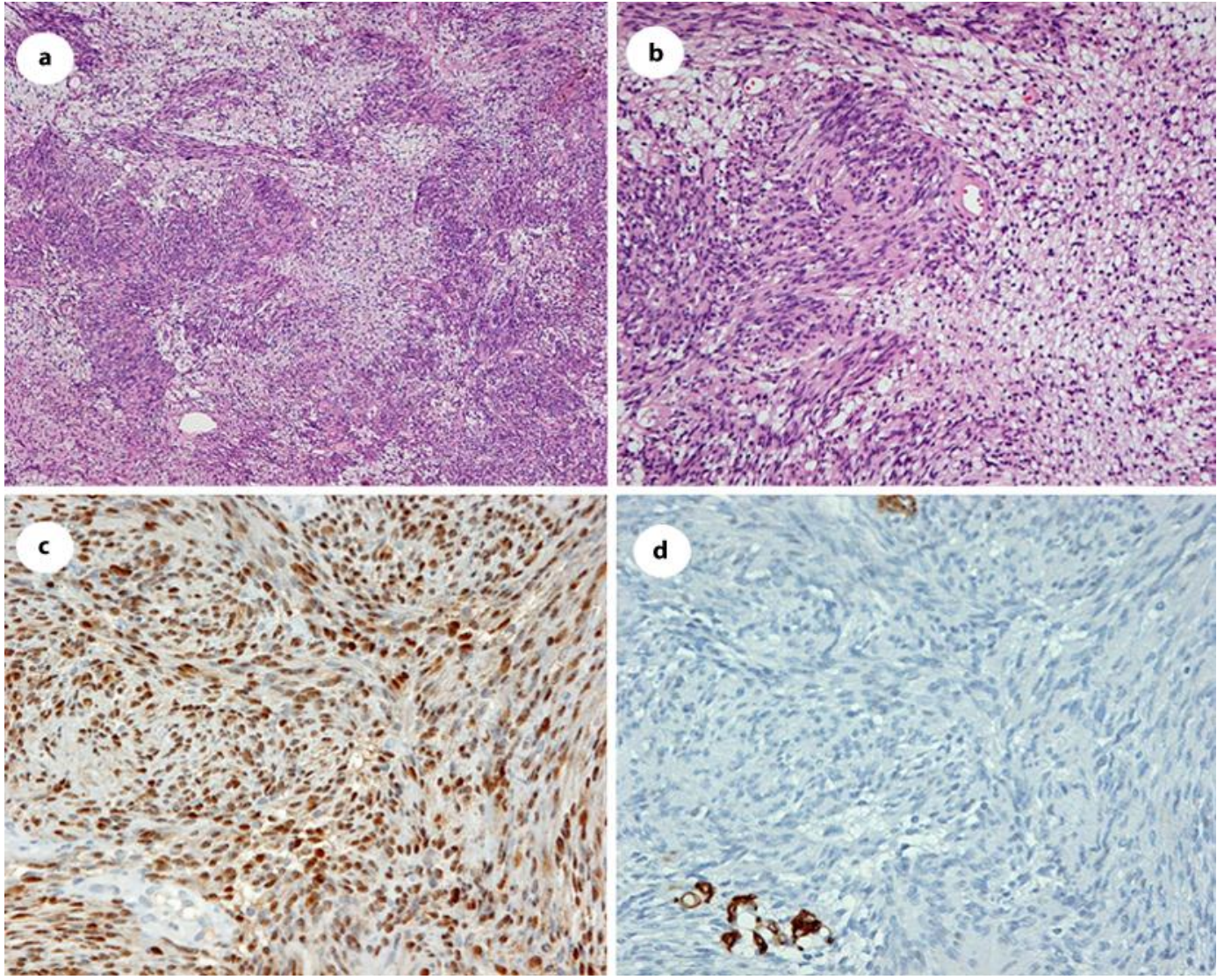

Fig. 3. Microscopic features of the schwannomas. The upper panel shows spindle cells in a fascicular pattern, and other regions in both tumors were composed of hypocellular spindle cells in a loose myxoid matrix (a, b; HE stain). The lower left panel shows that spindle cells reacted to S-100 (c). The lower right panel shows that spindle cells did not react to anti-smooth-muscle actin (d).

\section{References}

1 Scanlan DB: Primary retroperitoneal tumors. J Urol 1959;81:740-745.

-2 Das Gupta TK, Brasfield RD, Strong EW, Hajdu SI: Benign solitary schwannomas (neurilemomas). Cancer 1969;24:355-366.

3 Whitaker WG, Droulias C: Benign encapsulated neurilemoma: a report of 76 cases. Am Surg 1976;42:675-678.

4 Patel JJ, Taylor I, Fleming S: Multiple primary retroperitoneal tumours: a unique case. Br J Surg 1989;76:819-820.

5 Hurley L, Smith JJ 3rd, Larsen CR, Silverman ML: Multiple retroperitoneal schwannomas: case report and review of the literature. J Urol 1994;151:413-416.

6 Li Q, Gao C, Juzi JT, Hao X: Analysis of 82 cases of retroperitoneal schwannoma. ANZ J Surg 2007;77:237240.

7 Goh BK, Tan YM, Chung YF, Chow PK, Ooi LL, Wong WK: Retroperitoneal schwannoma. Am J Surg 2006;192:14-18.

8 Kinoshita T, Naganuma H, Ishii K, Itoh H: CT features of retroperitoneal neurilemmoma. Eur J Radiol 1998;27:67-71.

9 Hughes MJ, Thomas JM, Fisher C, Moskovic EC: Imaging features of retroperitoneal and pelvic schwannomas. Clin Radiol 2005;60:886-893. 
10 Kim SH, Choi BI, Han MC, Kim YI: Retroperitoneal neurilemoma: CT and MR findings. AJR Am J Roentgenol 1992;159:1023-1026.

11 Allolio B, Hahner S, Weismann D, Fassnacht M: Management of adrenocortical carcinoma. Clin Endocrinol (Oxf) 2004;60:273-287.

12 Francis IR, Gross MD, Shapiro B, Korobkin M, Quint LE: Integrated imaging of adrenal disease. Radiology 1992;184:1-13.

13 Inokuchi T, Takiuchi H, Moriwaki Y, Ka T, Takahashi S, Tsutsumi Z, Shima H, Hirota S, Yamamoto T: Retroperitoneal ancient schwannoma presenting as an adrenal incidentaloma: CT and MR findings. Magn Reson Imaging 2006;24:1389-1393.

14 Friberg S, Mattson S: On the growth rates of human malignant tumors: implications for medical decision making. J Surg Oncol 1997;65:284-297.

15 Mohyuddin A, Vokurka EA, Evans DG, Ramsden RT, Jackson A: Is clinical growth index a reliable predictor of tumour growth in vestibular schwannomas? Clin Otolaryngol Allied Sci 2003;28:85-90. 